\title{
Rassegna giurisprudenziale
}

\author{
(a cura di Nicola Lucifero)
}

\section{$\underline{\text { AGRICOLTURA }}$}

T.A.R. Lazio Roma, Sez. I bis, 06/05/2021, n. 5298

Terreno demaniale - pascolo - concessione - preferenza imprenditoria giovanile Inizio modulo

Fine modulo

In un procedura per l'assegnazione di una concessione su un terreno demaniale per il pascolo e lo sfalcio dell'erba deve essere data prevalenza al soggetto che vanti un diritto di preferenza in favore c.d. giovane imprenditore agricolo ai sensi dell'art. 6, comma 4-bis, $d$. lgs. 18 maggio 2001, n. 228, piuttosto che al soggetto che intenda esercitare un diritto di prelazione agraria ex all'art. 4-bis della legge 3 maggio 1982, n. 203.

CGUE, 08/07/2021, C-830/19, C.J. contro Région wallonne

Rinvio pregiudiziale - Agricoltura - Fondo europeo agricolo per lo sviluppo rurale (FEASR) - Regolamento (UE) n. 1305/2013 - Regolamento delegato (UE) n. 807/2014 - Insediamento dei giovani agricoltori - Sviluppo delle aziende agricole - Aiuti all'avviamento d'impresa per giovani agricoltori Condizioni d'accesso - Equivalenza - Insediamento in qualità di capo non unico dell'azienda - Massimali - Fissazione - Criteri - Produzione standard dell'azienda agricola

Gli articoli 2, 5 e 19 del regolamento (UE) n. 1305/2013 del Parlamento europeo e del Consiglio, del 17 dicembre 2013, sul sostegno allo sviluppo rurale da parte del Fondo europeo agricolo per lo sviluppo rurale (FEASR) e che abroga il regolamento (CE) n. 1698/2005 del Consiglio, in combinato disposto con gli articoli 2 e 5 del regolamento delegato (UE) n. 807/2014 della Commissione, dell'11 marzo 2014, che integra talune disposizioni del regolamento (UE) n. 1305/2013 del Parlamento europeo e del Consiglio sul sostegno allo sviluppo rurale da parte del Fondo europeo agricolo per lo sviluppo rurale (FEASR) e che introduce disposizioni transitorie, devono essere interpretati nel senso che non ostano a una normativa nazionale in forza della quale il criterio di determinazione del massimale che consente ad un giovane agricoltore, che si insedia in qualità di capo non unico dell'azienda, di accedere agli aiuti all'avviamento d'impresa, è quello della produzione lorda standard dell'intera azienda agricola, e non soltanto della quota di tale giovane agricoltore in tale azienda.

CGUE, 15/04/2021, C-736/19, ZS "Plaukti"

Rinvio pregiudiziale - Agricoltura - Fondo europeo agricolo per lo sviluppo rurale (FEASR) - Regolamento (CE) n. 1698/2005 - Regolamento (UE) n. 65/2011 Articolo 16, paragrafo 5, terzo comma - Regolamento (CE) n. 73/2009 - Articoli 4 
e 6 - Regolamento (CE) n. 1122/2009 - Sostegno dello sviluppo rurale - Pagamenti agroambientali - Sostegno per il mantenimento della biodiversità nelle praterie - Inosservanza dei presupposti per la concessione di tali pagamenti - Sfalcio prematuro - Riduzione ed esclusione di detti pagamenti - Norme obbligatorie - Criteri di gestione obbligatori - Requisiti minimi per le buone condizioni agronomiche e ambientali - Impegni che vanno al di là delle norme obbligatorie, dei requisiti minimi e di altre specifiche norme obbligatorie prescritte dalla legislazione nazionale

1) L'articolo 16, paragrafo 5, terzo comma, del regolamento (UE) n. 65/2011 della Commissione, del 27 gennaio 2011, che stabilisce modalità di applicazione del regolamento (CE) n. 1698/2005 del Consiglio per quanto riguarda l'attuazione delle procedure di controllo e della condizionalità per le misure di sostegno dello sviluppo rurale, dev'essere interpretato nel senso che non è applicabile nel caso in cui il richiedente il sostegno non abbia rispettato gli impegni agroambientali relativi ai requisiti riguardanti lo sfalcio, senza che sia stata constatata alcuna modifica del gruppo di colture interessato.

2) Gli articoli 4 e 6 del regolamento (CE) n. 73/2009 del Consiglio, del 19 gennaio 2009, che stabilisce norme comuni relative ai regimi di sostegno diretto agli agricoltori nell'ambito della politica agricola comune e istituisce taluni regimi di sostegno a favore degli agricoltori, e che modifica i regolamenti (CE) n. 1290/2005, (CE) n. 247/2006, (CE) n. $378 / 2007$ e abroga il regolamento (CE) n. 1782/2003, in combinato disposto con l'articolo 39, paragrafo 3, del regolamento (CE) n. 1698/2005 del Consiglio, del 20 settembre 2005, sul sostegno allo sviluppo rurale da parte del Fondo europeo agricolo per lo sviluppo rurale (FEASR), devono essere interpretati nel senso che essi ostano a una normativa nazionale secondo la quale un medesimo requisito può al contempo costituire un requisito minimo in materia di buone condizioni agronomiche e ambientali e un requisito che va al di là di tali requisiti minimi, ossia un presupposto per la concessione di pagamenti agroambientali.

CGUE, 10/03/2021, C-365/19, FD contro Staatliches Amt für Landwirtschaft und Umwelt Mittleres Mecklenburg

Rinvio pregiudiziale - Politica agricola comune - Pagamenti diretti - Regolamento (UE) n. 1307/2013 - Articolo 24 - Giovane agricoltore che ha beneficiato di una prima assegnazione di diritti all' aiuto - Articolo 30, paragrafo 6 - Regolamento delegato (UE) n. 639/2014 - Articolo 28, paragrafo 2 - Assegnazione supplementare di diritti all'aiuto a partire dalla riserva nazionale

L'articolo 30, paragrafo 6, del regolamento (UE) n. 1307/2013 del Parlamento europeo $e$ del Consiglio, del 17 dicembre 2013, recante norme sui pagamenti diretti agli agricoltori nell'ambito dei regimi di sostegno previsti dalla politica agricola comune e che abroga il regolamento (CE) n. 637/2008 del Consiglio e il regolamento (CE) n. 73/2009 del Consiglio, in combinato disposto con l'articolo 28, paragrafo 2, del regolamento delegato (UE) n. 639/2014 della Commissione, dell'11 marzo 2014, che integra il regolamento $n$. $1307 / 2013$ e che modifica l'allegato $X$ di tale regolamento, deve essere interpretato nel senso che un giovane agricoltore, ai sensi dell'articolo 30, paragrafo 11, lettera a), del regolamento n. 1307/2013, in combinato disposto con l'articolo 50, paragrafo 2, di detto regolamento, che abbia già beneficiato, ai sensi dell'articolo 24 del medesimo regolamento, di una prima assegnazione di diritti all'aiuto pari al massimo al numero degli ettari ammissibili 
che ha dichiarato al momento della sua domanda, ha diritto a ricevere, successivamente, un'assegnazione supplementare di diritti all'aiuto a partire dalla riserva nazionale pari al numero aggiuntivo di ettari ammissibili che detiene in quel momento e per $i$ quali non detiene alcun diritto all'aiuto. Tale diritto è subordinato all'esistenza di fondi disponibili in misura sufficiente nelle riserve nazionale o regionali. In caso contrario, l'assegnazione dovrà avvenire in modo da assicurare la parità di trattamento tra gli agricoltori ammissibili ai diritti ai sensi dell'articolo 30, paragrafo 6, del regolamento n. 1307/2013 e da evitare distorsioni del mercato e della concorrenza.

CGUE, 4/02/2021, C-640/19

Rinvio pregiudiziale - Agricoltura - Organizzazione comune dei mercati Regolamento (CE) n. 1234/2007 - Quote latte - Prelievi sulle eccedenze - Latte rivolto alla produzione di formaggi che beneficiano di una denominazione d'origine protetta (DOP) e sono destinati all'esportazione verso paesi terzi - Esclusione - Articolo 32, lettera a), articolo 39, paragrafi 1 e 2, lettera a), articolo 40, paragrafo 2, e articolo 41, lettera b), TFUE - Principi di proporzionalità e di non discriminazione - Validità

1) Gli articoli 55, 65 e 78 del regolamento (CE) n. 1234/2007 del Consiglio, del 22 ottobre 2007, recante organizzazione comune dei mercati agricoli e disposizioni specifiche per taluni prodotti agricoli, come modificato dal regolamento (CE) n. 248/2008 del Consiglio, del 17 marzo 2008, devono essere interpretati nel senso che essi non escludono dal calcolo delle quote nazionali per la produzione di latte e di altri prodotti lattiero-caseari, nonché dal calcolo dei prelievi sulle eccedenze, $i$ quantitativi di latte rivolti alla produzione di formaggi che beneficiano di una denominazione d'origine protetta e sono destinati ad essere esportati verso paesi terzi.

2) L'esame della terza questione pregiudiziale non ha rivelato alcun elemento idoneo ad inficiare la validità degli articoli 55, 65 e 78 del regolamento n. 1234/2007, come modificato dal regolamento n. 248/2008.

CGUE, 27 gennaio 2021, C-361/19, De Ruiter vof contro Minister van Landbouw, Natuur en Voedselkwaliteit

Rinvio pregiudiziale - Politica agricola comune - Regime di sostegno agli agricoltori - Regolamento (UE) n. 1306/2013 - Articolo 97, paragrafo 1, e articolo 99, paragrafo 1 - Pagamenti diretti - Riduzioni ed esclusioni in caso di inosservanza delle regole di condizionalità - Determinazione dell'anno da prendere in considerazione ai fini del calcolo della percentuale di riduzione - Sanzioni proporzionate, effettive e dissuasive - Regolamento di esecuzione (UE) n. 809/2014 Articolo 73, paragrafo 4, primo comma, lettera a)

L'articolo 97, paragrafo 1, primo comma, e l'articolo 99, paragrafo 1, primo comma, del regolamento (UE) n. 1306/2013 del Parlamento europeo e del Consiglio, del 17 dicembre 2013, sul finanziamento, sulla gestione e sul monitoraggio della politica agricola comune e che abroga i regolamenti del Consiglio (CEE) n. 352/78, (CE) n. 165/94, (CE) n. 2799/98, (CE) n. 814/2000, (CE) n. 1290/2005 e (CE) n. 485/2008, nonché l'articolo 73, paragrafo 4, primo comma, lettera a), del regolamento di esecuzione (UE) n. 809/2014 della Commissione, del 17 luglio 2014, recante modalità di applicazione del regolamento n. 1306/2013 
per quanto riguarda il sistema integrato di gestione e di controllo, le misure di sviluppo rurale e la condizionalità, devono essere interpretati nel senso che le riduzioni dei pagamenti diretti per inosservanza delle regole di condizionalità devono essere calcolate sulla base dei pagamenti corrisposti o da corrispondere per l'anno in cui si è verificata tale inosservanza.

\section{$\underline{\text { ALIMENTI }}$}

CGUE, 15/04/2021, C-53/20, Hengstenberg GmbH \& Co. KG contro Spreewaldverein $\mathrm{eV}$

Rinvio pregiudiziale - Protezione delle indicazioni geografiche e delle denominazioni d'origine dei prodotti agricoli ed alimentari - Regolamento (UE) n. 1151/2012 - Articolo 49, paragrafo 3, primo comma e paragrafo 4, secondo comma - Articolo 53, paragrafo 2, primo comma - Modifica del disciplinare di un prodotto - Cetriolini della Foresta della Sprea (Germania) "Spreewälder Gurken (IGP)" - Modifiche non minori - Opposizione - Dichiarazione di opposizione alla domanda di modifica - Ricorso contro la decisione che accoglie tale domanda - Nozione di "interesse legittimo"

L'articolo 49, paragrafo 3, primo comma, e paragrafo 4, secondo comma, del regolamento (UE) n. 1151/2012, del Parlamento europeo e del Consiglio, del 21 novembre 2012, sui regimi di qualità dei prodotti agricoli e alimentari, in combinato disposto con l'articolo 53, paragrafo 2, primo comma, di quest'ultimo, deve essere interpretato nel senso che, nell'ambito della procedura applicabile alle domande di modifica non minore del disciplinare di un prodotto che beneficia di un'indicazione geografica protetta, ogni persona fisica o giuridica che subisca un pregiudizio economico, reale o potenziale, però non del tutto inverosimile, dalle modifiche richieste può vantare l'«interesse legittimo» richiesto per dichiarare un'opposizione alla domanda di modifica presentata o per proporre ricorso contro la decisione che accoglie detta domanda, qualora il rischio di pregiudizio agli interessi di tale persona non sia puramente improbabile o ipotetico, circostanza che spetta al giudice del rinvio verificare.

Corte cost., 09/03/2021, n. 31

Alimenti - Definizione e promozione dell'utilizzo, nelle mense scolastiche, dei prodotti a chilometro zero e da filiera corta selezionati in base al collegamento con il territorio regionale - Violazione della competenza esclusiva statale in materia di tutela della concorrenza e della libera circolazione dei prodotti nel territorio nazionale - Illegittimità costituzionale

Sono dichiarati costituzionalmente illegittimi - per violazione degli artt. 117, secondo comma, lett. e), e 120 Cost. - gli artt. 2, 3 e 4 della legge reg. Toscana n. 75 del 2019, che definiscono e promuovono l'utilizzo, nelle mense scolastiche, dei prodotti a chilometro zero e da filiera corta selezionati in base al collegamento con il territorio regionale. Le norme impugnate dal Governo, pur perseguendo il fine di valorizzare i prodotti del territorio - di per sé non illegittimo - realizzano siffatto obiettivo favorendo i prodotti e i produttori regionali, con una evidente discriminazione per chi faccia uso di prodotti di diversa provenienza. Infatti, in difformità da quanto previsto dalla normativa statale, le definizioni dei prodotti a 
chilometro zero e da filiera corta non si collegano, in quanto tali, a un criterio di prossimità tra produzione e vendita, né a un trasporto delle merci breve o con una bassa emissione di sostanze inquinanti, ma fanno una selezione in base al collegamento con il territorio regionale. In tal modo le disposizioni impugnate non solo non favoriscono la concorrenza, ma chiaramente la alterano; il che si risolve altresi in un ostacolo alla libera circolazione delle merci. La declaratoria d'illegittimità costituzionale deve estendersi anche all'art. 2, comma 3 - che si limita a far rientrare i prodotti a denominazione protetta o a marchio tutelato (oggetto, tra l'altro, di specifiche disposizioni di diritto europeo), ove ne rispettino $i$ requisiti, nelle definizioni di chilometro zero e filiera corta - che, in virtù del rapporto di stretta concatenazione oggettiva e funzionale con le censurate disposizioni resta privo di autonoma portata normativa. Le procedure di selezione dei concorrenti e $i$ criteri di aggiudicazione degli appalti pubblici sono ascrivibili alla materia della tutela della concorrenza di cui all'art. 117, secondo comma, lett. e), Cost. - che riflette la definizione operante in ambito comunitario - nella specie alla concorrenza «per il mercato»

\section{$\underline{\text { AMBIENTE }}$}

CGUE, 12/05/2021, C-87/20, Hauptzollamt B contro XY

Protezione di specie della flora e della fauna selvatiche mediante il controllo del loro commercio - Regolamenti (CE) nn. 338/97 e 865/2006 - Caviale delle specie di storione - Introduzione nel territorio doganale dell'Unione europea a titolo di oggetti personali o domestici - Licenza di importazione - Deroga - Quantitativo massimo di 125 grammi per persona - Superamento - Intenzione di donare a terzi

1) L'articolo 7, paragrafo 3, del regolamento (CE) n. 338/97 del Consiglio, del 9 dicembre 1996, relativo alla protezione di specie della flora e della fauna selvatiche mediante il controllo del loro commercio, come modificato dal regolamento (UE) n. 1320/2014 della Commissione, del $1^{\circ}$ dicembre 2014, deve essere interpretato nel senso che il caviale delle specie di storione, al momento della sua introduzione nel territorio doganale dell'Unione europea, può essere considerato un «oggetto personale o domestico» ai sensi di tale disposizione, qualora sia destinato a essere regalato a terzi, purché da nessun elemento emerga una finalità commerciale, e può pertanto beneficiare della deroga, prevista da detta disposizione, all'obbligo per il suo importatore di presentare una licenza di importazione.

2) L'articolo 57, paragrafo 5, lettera a), del regolamento (CE) n. 865/2006 della Commissione, del 4 maggio 2006, recante modalità di applicazione del regolamento n. 338/97, come modificato dal regolamento (UE) 2015/870 della Commissione, del 5 giugno 2015, deve essere interpretato nel senso che, ove il quantitativo di caviale delle specie di storione introdotto nel territorio doganale dell'Unione europea superi il quantitativo massimo di 125 grammi per persona e l'importatore non sia in possesso di una licenza rilasciata ai fini dell'importazione effettuata, l'intero quantitativo di caviale delle specie di storione in tal modo importato dovrà essere confiscato dall'autorità doganale competente.

CGUE, 29/04/2021, C-617/19, Granarolo SpA contro Ministero dell'Ambiente e della Tutela del Territorio e del Mare e a. 
Rinvio pregiudiziale - Ambiente - Direttiva 2003/87/CE - Sistema per lo scambio di quote di emissioni dei gas a effetto serra - Articolo 3, lettera e) - Nozione di "impianto" - Articolo 3, lettera f) - Nozione di "gestore" - Allegato I, punti 2 e 3 - Regola dell'aggregazione - Somma delle capacità delle attività di un impianto - Cessione di un' unità di cogenerazione di energia elettrica e calore da parte del proprietario di uno stabilimento industriale - Contratto di fornitura di energia tra le imprese cedente e cessionaria - Aggiornamento dell'autorizzazione ad emettere gas a effetto serra

L'articolo 3, lettere e) e f), della direttiva 2003/87/CE del Parlamento europeo e del Consiglio, del 13 ottobre 2003, che istituisce un sistema per lo scambio di quote di emissioni dei gas a effetto serra nella Comunità e che modifica la direttiva 96/61/CE del Consiglio, come modificata dalla direttiva 2009/29/CE del Parlamento europeo e del Consiglio, del 23 aprile 2009, in combinato disposto con i punti 2 e 3 dell'allegato I della stessa, deve essere interpretato nel senso che esso non osta a che il proprietario di uno stabilimento produttivo dotato di una centrale termica la cui attività rientra nell'ambito di applicazione di tale allegato I possa ottenere un aggiornamento della sua autorizzazione ad emettere gas a effetto serra, ai sensi dell'articolo 7 di tale direttiva, se ha ceduto un'unità di cogenerazione situata nello stesso sito industriale di tale stabilimento ed esercente un'attività con una capacità inferiore alla soglia stabilita in detto allegato I ad un'impresa specializzata nel settore dell'energia, concludendo con tale impresa un contratto che prevede, in particolare, la fornitura a detto stabilimento dell'energia prodotta da tale unità di cogenerazione, sempre che la centrale termica e l'unità di cogenerazione non costituiscano un solo ed unico impianto, ai sensi dell'articolo 3, lettera e), di detta direttiva, e che, in ogni caso, il proprietario dello stabilimento produttivo non sia più il gestore dell'unità di cogenerazione, ai sensi dell'articolo 3, lettera f), della medesima direttiva

CGUE, 04/03/2021, C-473-474/19, Föreningen Skydda Skogen e a. contro Länsstyrelsen i Västra Götalands län e a.

Rinvio pregiudiziale - Ambiente - Direttiva 92/43/CEE - Conservazione degli habitat naturali e della flora e della fauna selvatiche - Articolo 12, paragrafo 1 Direttiva 2009/147/CE - Conservazione degli uccelli selvatici - Articolo 5 - Silvicoltura - Divieti diretti a garantire la conservazione delle specie protette - Progetto di disboscamento definitivo - Sito ospitante specie protette

1) L'articolo 5 della direttiva 2009/147/CE del Parlamento europeo e del Consiglio, del 30 novembre 2009, concernente la conservazione degli uccelli selvatici, dev'essere interpretato nel senso che osta ad una prassi nazionale in base alla quale $i$ divieti previsti da tale disposizione riguardino unicamente le specie elencate nell'allegato I di tale direttiva, quelle minacciate ad un certo livello o la cui popolazione presenti una tendenza alla diminuzione a lungo termine.

2) L'articolo 12, paragrafo 1, lettere da a) a c), della direttiva 92/43/CEE del Consiglio, del 21 maggio 1992, relativa alla conservazione degli habitat naturali e seminaturali e della flora e della fauna selvatiche, dev'essere interpretato nel senso che, da un lato, osta ad una prassi nazionale secondo la quale, laddove l'oggetto di un'attività umana, quale la silvicoltura o l'utilizzazione del territorio, sia manifestamente diverso dall'uccisione o dalla perturbazione di specie animali, $i$ divieti previsti da tale disposizione si applichino unicamente 
a condizione che sussista un rischio di impatto negativo sullo stato di conservazione delle specie interessate e, dall'altro, la protezione offerta da tale disposizione non cessa di applicarsi alle specie che hanno raggiunto uno stato di conservazione soddisfacente.

3) L'articolo 12, paragrafo 1, lettera d), della direttiva 92/43 dev'essere interpretato nel senso che osta ad una prassi nazionale secondo la quale, nel caso in cui la permanenza della funzionalità ecologica dell'habitat naturale della specie interessata in una determinata zona sia, nonostante le precauzioni prese, perduto per deterioramento, distruzione o degradazione, direttamente o indirettamente, per effetto dell'attività in questione considerata isolatamente o cumulativamente con altre, il divieto previsto dalla disposizione suddetta non operi fino a quando lo stato di conservazione della specie in questione rischi di deteriorarsi.

CGUE, 15 aprile 2021, C-470/19, Friends of the Irish Environment Ltd contro Commissioner for Environmental Information

Rinvio pregiudiziale - Convenzione di Aarhus - Direttiva 2003/4/CE - Diritto di accesso alle informazioni ambientali detenute dalle autorità pubbliche - Articolo 2, punto 2 - Nozione di "autorità pubblica" - Organi o istituzioni che agiscono nell'esercizio del potere giudiziario - Informazioni contenute nel fascicolo di un procedimento giurisdizionale chiuso

L'articolo 2, punto 2, della direttiva 2003/4/CE del Parlamento europeo e del Consiglio, del 28 gennaio 2003, sull'accesso del pubblico all'informazione ambientale e che abroga la direttiva 90/313/CEE del Consiglio, deve essere interpretato nel senso che esso non disciplina l'accesso alle informazioni ambientali contenute nei fascicoli giudiziari, nei limiti in cui gli organi giurisdizionali o le istituzioni poste sotto il loro controllo, e che presentano quindi stretti legami con questi ultimi, non costituiscono «autorità pubbliche» ai sensi di tale disposizione e non rientrano dunque nell'ambito di applicazione di tale direttiva.

CGUE, 14 gennaio 2021, C-826/18, LB e a. contro College van burgemeester en wethouders van de gemeente Echt-Susteren

Rinvio pregiudiziale - Convenzione di Aarhus - Articolo 9, paragrafi 2 e 3 Accesso alla giustizia - Mancato accesso alla giustizia per il pubblico diverso dal pubblico interessato - Ricevibilità del ricorso subordinata alla previa partecipazione al processo decisionale.

1) L'articolo 9, paragrafo 2, della convenzione sull'accesso alle informazioni, la partecipazione del pubblico ai processi decisionali e l'accesso alla giustizia in materia ambientale, firmata ad Aarhus (Danimarca) il 25 giugno 1998 e approvata a nome della Comunità europea con la decisione 2005/370/CE del Consiglio, del 17 febbraio 2005, deve essere interpretato nel senso che esso non osta a che $i$ membri del «pubblico» di cui all'articolo 2, paragrafo 4, di tale convenzione non abbiano accesso in quanto tali alla giustizia, al fine di impugnare una decisione rientrante nell'ambito di applicazione dell'articolo 6 della medesima. Per contro, l'articolo 9, paragrafo 3, di detta convenzione osta a che tali persone non possano avere accesso alla giustizia per avvalersi di più ampi diritti di partecipazione al processo decisionale, che siano loro conferiti unicamente dal diritto ambientale nazionale di uno Stato membro.

2) L'articolo 9, paragrafo 2, della convenzione sull'accesso alle informazioni, la partecipazione del pubblico ai processi decisionali e l'accesso alla giustizia in materia ambientale, fir- 
mata ad Aarhus il 25 giugno 1998 e approvata a nome della Comunità europea con la decisione 2005/370, deve essere interpretato nel senso che osta a che la ricevibilità dei ricorsi giurisdizionali a cui esso si riferisce, esperiti da organizzazioni non governative facenti parte del «pubblico interessato», di cui all'articolo 2, paragrafo 5, di tale convenzione, sia subordinata alla partecipazione di tali organizzazioni alla procedura di preparazione relativa alla decisione impugnata, anche se tale condizione non si applica qualora non possa essere loro ragionevolmente addebitato di non avervi partecipato. Per contro, l'articolo 9, paragrafo 3, di detta convenzione non osta a che la ricevibilità di un ricorso giurisdizionale a cui esso si riferisce sia subordinata alla partecipazione del ricorrente alla procedura di preparazione relativa alla decisione impugnata a meno che, tenuto conto delle circostanze del caso, il fatto di non essere intervenuto in tale procedura non gli possa essere ragionevolmente addebitato.

\section{BOSCHI E FORESTE}

Cass. pen., Sez. III, 3/03/2021, n. 8499

Bellezze naturali - Alberi - Abbattimento di alberi di alto fusto in area sottoposta a vincolo paesaggistico in assenza o in difformità della prescritta autorizzazione - Reato di deturpamento delle bellezze naturali di cui all'art. 734 c.p.

L'abbattimento di alberi in difetto della preventiva autorizzazione paesaggistica configura il reato di cui all'art. 181, d.lgs. $n .42$ del 2004, in quanto attività idonea a compromettere $i$ valori ambientali incidendo in modo apprezzabile sull'assetto del territorio. Commette il reato di deturpa-mento delle bellezze naturali di cui all'art. 734 c.p. chi effettui l'abbattimento di alberi di alto fusto in area sottoposta a vincolo paesaggistico in assenza o in difformità della prescritta autorizzazione

T.A.R. Puglia Bari, Sez. III, 06/04/2021, n. 577

Boschi e foreste - vincolo paesaggistico - recepimento in atti di pianificazione regionale

In tema di vincolo boschivo, lo stesso in quanto rilevante ex lege prescinde dal suo effettivo recepimento negli atti di pianificazione generale e/o dalla sua rappresentazione cartografica nella pianificazione paesaggistica, che al riguardo non interviene con effetti costituitivi limitandosi ad operare una mera ricognizione circa l'effettiva esistenza del bene tutelato in base alle sue qualità intrinseche.

\section{$\underline{\text { BONIFICA }}$}

Cass. civ., Sez. Unite, 26/02/2021, n. 5422

Opere idrauliche - progettazione e realizzazione - affidamento a consorzi di bonifica - gestione dell'acqua - responsabilità ex art. 2051 c.c. - presupposti

Considerato che le funzioni di progettazione, realizzazione e gestione delle opere idrauliche, nonché $i$ conseguenti poteri di custodia, possono essere dalle Regioni delegate ai consorzi di bonifica o ai concessionari delle relative opere, la cui posizione, quindi, deve essere valu- 
tata di volta in volta sulla base delle singole legislazioni regionali (tenendo presente quanto stabilito a livello statale in tema di classificazione delle opere idrauliche e riparto dei relativi compiti di gestione e manutenzione, nonché di esecuzione delle opere di attuazione del piano generale di bonifica), occorre verificare se ed in quale misura il consorzio di bonifica sia, nel caso concreto, realmente investito di funzioni di manutenzione dei corsi d'acqua, con conseguente insorgenza di responsabilità a titolo di custodia, ai sensi dell'art. 2051 cod. civ. Ciò posto, l'inserimento espresso di un bacino (di cui fa parte l'alveo la cui esondazione, a causa di forti piogge, ha recato danni ad un provato) nel territorio del Consorzio, da un lato, esclude la necessità di un apposito atto di consegna da parte della Regione e, dall'altro, attribuisce all'ente compiti di manutenzione, restando irrilevante verificare se, in concreto, il Consorzio abbia o meno proceduto alla predisposizione di un piano di classifica e di contribuzione consortile, esigendone il pagamento.

\section{CACCIA E PESCA}

CGUE, Sez. I, 17/03/2021, n. 900/19

Rinvio pregiudiziale - Ambiente - Direttiva 2009/147/CE - Conservazione degli uccelli selvatici - Articoli 5 e 8 - Divieto di ricorrere a qualsiasi metodo di cattura degli uccelli - Articolo 9, paragrafo 1 - Autorizzazione a ricorrere in virtù di una deroga a un siffatto metodo consacrato dagli usi tradizionali - Presupposti Assenza di altra soluzione soddisfacente - Giustificazione dell'assenza di "altra soluzione soddisfacente" con la sola preservazione di detto metodo tradizionale - Selettività delle catture - Normativa nazionale che autorizza la cattura di uccelli tramite l'impiego di vischio

1) L'articolo 9, paragrafi 1 e 2, della direttiva 2009/147/CE del Parlamento europeo e del Consiglio, del 30 novembre 2009, concernente la conservazione degli uccelli selvatici, deve essere interpretato nel senso che il carattere tradizionale di un metodo di cattura di uccelli non è sufficiente, di per sé, a dimostrare che un'altra soluzione soddisfacente, ai sensi di tale disposizione, non possa sostituirsi a detto metodo.

2) L'articolo 9, paragrafo 1, lettera c), della direttiva 2009/147 deve essere interpretato nel senso che esso osta a una normativa nazionale che autorizza, in deroga all'articolo 8 di tale direttiva, un metodo di cattura che comporta catture accessorie, qualora queste ultime, pur essendo di volume esiguo e di una durata limitata, siano idonee ad arrecare alle specie non bersaglio catturate danni che non siano trascurabili.

Inizio modulo

Fine modulo

Corte cost., 06/07/2021, n. 138

Caccia - appostamenti temporanei su fondi altrui - consenso implicito del proprietario - competenza statale

E fondata la questione di legittimità costituzionale sollevata in relazione all'art. 2, comma 1, 6 e 9 della legge della Regione Liguria 19 maggio 2020, n. 9 che ha aggiunto, nella parte finale dell'art. 29, comma 13, della legge della Regione Liguria $1^{\circ}$ luglio 1994, $n$. 29 (Norme regionali per la protezione della fauna omeoterma e per il prelievo venatorio), 
un ulteriore periodo ai sensi del quale "il consenso si intende validamente accordato nel caso in cui non esiste un formale diniego". La disposizione impugnata è ritenuta in contrasto con la riserva di competenza legislativa allo Stato in materia di «ordinamento civile» di cui all'art. 117, secondo comma, lett. l), Cost. in quanto, consentendo ai cacciatori di mantenere, se il proprietario non manifesta espressamente il suo dissenso, sul fondo altrui il materiale utilizzato per la costruzione degli appostamenti temporanei, inciderebbe sulle facoltà dominicali garantite dall'art. 832 c.c. Invero la disciplina del diritto di proprietà attiene alla competenza esclusiva dello Stato in materia di ordinamento civile e l'ordinamento del diritto privato si pone quale limite alla legislazione regionale, in quanto fondato sull'esigenza, sottesa al principio costituzionale di eguaglianza, di garantire sul territorio nazionale l'uniformità della disciplina dettata per i rapporti tra privati. Il limite dell'ordinamento civile, quindi, identifica un'area riservata alla competenza esclusiva della legislazione statale e comprende $i$ rapporti tradizionalmente oggetto di codificazione. Nel caso in esame, la norma impugnata nell'aggiungere nella parte finale dell'art. 29, un ulteriore periodo, introduce una presunzione di consenso del proprietario del fondo al mantenimento su di esso del materiale usato per la costruzione degli appostamenti temporanei, che eccede $i$ limiti del legittimo intervento del legislatore regionale, invadendo la competenza riservata allo Stato nella materia "ordinamento civile".

Corte cost., 03/06/2021, n. 116

Piani di abbattimento della fauna - partecipazione dei cacciatori - corsi di formazione - approvazione del ISPRA

È costituzionalmente illegittimo l'art. 1, comma 1, lettera a), della legge della Regione Sardegna 27 febbraio 2020, n. 5 (Modifiche all'articolo 6 della legge regionale n. 23 del 1998 in materia di piani di abbattimento), che sostituisce l'art. 6, comma 1, lettera f), della legge della Regione Sardegna 29 luglio 1998, n. 23 (Norme per la protezione della fauna selvatica e per l'esercizio della caccia in Sardegna), nella parte in cui non prevede che $i$ corsi di formazione specifici, ai quali devono aver partecipato i meri cacciatori delegati dai proprietari e conduttori dei fondi su cui vanno attuati i piani di abbattimento della fauna selvatica, siano concordati con l'Istituto superiore per la protezione e la ricerca ambientale (ISPRA). Il controllo della fauna selvatica è demandato alle Regioni, ai sensi dell'art. 19 della legge n. 157 del 1992 e deve essere espletato mediante il ricorso a metodi ecologici e, solo allorché l'Istituto nazionale per la fauna selvatica (oggi Istituto superiore per la protezione e la ricerca ambientale - ISPRA) abbia verificato l'inefficacia di tali metodi ecologici, le Regioni possono autorizzare piani di abbattimento. Orbene, con riferimento alla delegabilità degli abbattimenti a soggetti diversi dai proprietari e conduttori (muniti solo di un'autorizzazione alla caccia), i corsi di cui alla norma censurata non risultano pienamente idonei a legittimare l'ampliamento dell'elenco dei soggetti autorizzati agli abbattimenti, di cui all'art. 19 della legge n. 157 del 1992, in quanto non è previsto che i corsi in questione siano concordati con l'Istituto superiore per la protezione e la ricerca ambientale, con conseguente illegittimità costituzionale della norma in parola. 
Corte cost., 31/05/2021, n. 113

Caccia - controllo del prelievo venatorio - estensione del periodo per specie la cui presenza sul territorio sia divenuta eccessiva e critica - illegittimità

È costituzionalmente illegittimo l'art. 12, comma 5, lett. a), della legge della Regione Molise 30 aprile 2020,n. 1 (Legge di stabilità regionale 2020), che aggiunge il comma 1-bis all'art. 27 della legge della Regione Molise 10 agosto 1993, n. 19 (Norme per la protezione della fauna selvatica omeoterma e per il prelievo venatorio). Il legislatore regionale, nell'esercizio della propria competenza legislativa residuale in materia di caccia, ha, infatti, violato l'art. 117, comma 2, lett. s), Cost., perché ha ridotto il livello di protezione della fauna selvatica stabilito - mediante la definizione della durata dei periodi venatori dall'art. 18, commi 1 e 2, della legge n. 157 del 1992. Altresì, l'art. 27, comma 1-bis, della legge Reg. Molise n. 19 del 1993, introdotto dalla norma impugnata, attraverso l'indebita estensione dell'arco temporale del periodo del prelievo venatorio di determinate specie, la cui presenza sul territorio sia divenuta "eccessiva" e fonte di conseguenti "criticità", ha introdotto, nella sostanza, una surrettizia forma di controllo faunistico, svincolata però dai precisi limiti procedimentali previsti dal legislatore statale. Siffatta disciplina compromette pertanto il principio di gradualità e riduce lo standard minimo di tutela posto dal legislatore statale, cosi violando l'art. 117, secondo comma, lett. s), Cost., con conseguente declaratoria di incostituzionalità della norma censurata.

Corte cost., 17/02/2021, n. 21

Caccia - Norme della Regione Toscana - Interventi di tutela della produzione agricola e zootecnica - Affiancamento del personale regionale - Soggetti che abbiano frequentato appositi corsi di preparazione regionali sulla base di programmi concordati con $1^{\prime}$ ISPRA - Denunciata violazione della competenza esclusiva statale in materia di tutela dell'ambiente - Insussistenza - Non fondatezza della questione

Sono dichiarate non fondate le questioni di legittimità costituzionale - sollevate dal TAR Toscana in riferimento all'art. 117, secondo comma, lett. s), Cost. - dell'art. 37, comma 4, della legge reg. Toscana n. 3 del 1994, secondo cui per interventi di tutela della produzione agricola e zootecnica, compresi i piani di abbattimento, la Regione può affiancare al proprio personale anche soggetti che abbiano frequentato appositi corsi di preparazione organizzati dalla Regione stessa sulla base di programmi concordati con l'ISPRA. La norma censurata, integrando l'elenco di cui all'art. 19, comma 2, della legge n. 157 del 1992, incrementa lo standard di tutela ambientale fissato dalla disposizione statale. Il coinvolgimento dei cacciatori nei piani di abbattimento è infatti subordinato all'acquisizione di una specifica formazione, concordata con l'ISPRA, nonché sottoposto al coordinamento della polizia provinciale.

T.A.R. Valle d'Aosta Aosta, Sez. Unica, 09/03/2021, n. 16

Caccia - individuazione delle specie cacciabili - temporanee limitazioni

L'indicazione generale delle specie cacciabili è rimessa al legislatore nazionale e per la Valle d'Aosta al legislatore regionale munito in materia di potestà esclusiva (si veda l'art. 30 della L.R. n. 64 del 1994), ma ciò non esclude la possibilità di temporanee limitazioni al prelievo di alcune specie cacciabili adottabili con atto amministrativo, quale il calendario 
venatorio emesso con provvedimento regionale, il quale è un atto che ha un efficacia temporale limitata e che necessita di essere emanato ogni anno perché possono cambiare le circostanze concrete in cui è opportuno esercitare l'attività venatoria.

\section{CONTRATTI AGRARI}

Corte d'Appello Catanzaro, Sez. agraria, 18/03/2021

Contratti agrari - affitto - addizioni e miglioramenti - IAP - detrazione finanziamenti erogati

Ove rimanga accertata la qualifica di imprenditore agricolo a titolo principale dell'affittuario, a quest'ultimo non compete la detrazione dei finanziamenti erogati al fine della determinazione della misura dei miglioramenti, sul presupposto che la norma in discussione prevede che il suo computo è propriamente correlato alla configurazione di tale qualifica in capo alla parte affittuaria. Tanto viene spiegato dalla Corte territoriale sulla base di un'impostazione interpretativa, oltre che letterale, anche finalistica della controversa norma, siccome indirizzata, nel suo complesso, a favorire proprio chi esercita l'attività di impresa agricola a titolo principale.

\section{$\underline{\text { IMPOSTE, TASSE E CONTRIBUTI }}$}

Cons. Stato, Sez. III, 12/07/2021, n. 5281

Debiti - Registro nazionale AGEA - iscrizione - equiparazione iscrizione a ruolo.

L'iscrizione nel Registro Nazionale dei debiti di cui all'art. 8 ter, $1^{\circ}$ comma, della L. $n$. 33/2009, istituito presso AGEA, è equiparata all'iscrizione a ruolo delle somme dovute, ex art. 8 ter, $2^{\circ}$ comma, L. n. 33/2009.

Cass. civ., Sez. V, Ordinanza, 10/06/2021, n. 16210

Piccola proprietà contadina - acquisto - agevolazioni fiscali - recupero imposte - prescrizione

In tema di agevolazioni fiscali previste per l'acquisto di terreni agricoli al fine della formazione della piccola proprietà contadina, il termine triennale di prescrizione previsto dall'art. 4, comma terzo, della l. n. 604 del 1954 per il recupero delle imposte ordinarie, decorrente dalla scadenza del termine concesso per la presentazione del certificato definitivo recante l'attestazione del possesso dei requisiti di cui al precedente art. 2, si applica tanto nel caso in cui l'acquirente sia già in possesso della certificazione definitiva al momento della registrazione dell'acquisto, quanto nel caso in cui tale certificazione sia conseguita successivamente.

Cass. civ., Sez. V, Ordinanza, 13/05/2021, n. 12852

Piccola proprietà contadina - Agevolazioni tributarie ici - Terreni edificabili destinati ad uso agricolo - Riduzione della base imponibile - Presupposti - Impre nditore agricolo professionale (iap) - Terreni ubicati in regione diversa da quella che ha riconosciuto la qualifica di iap 
In tema di ICI, va esclusa l'agevolazione prevista dall'art. 2, comma 1, lett. b), d.lgs. $n$. 504 del 1992 per i terreni edificabili posseduti e condotti per l'esercizio di attività agricola da parte di imprenditore agricolo professionale, laddove i fondi siano ubicati in Regione diversa da quella che ha attribuito la qualifica di IAP, in quanto tale attribuzione tiene conto delle caratteristiche del singolo terreno regionale ai fini agricoli e, dunque, non può valere in modo generalizzato per l'intero territorio nazionale tenuto conto, altresì, che si è in presenza di norma di stretta interpretazione.

Cass. civ., Sez. V, Ordinanza, 12/02/2021, n. 3598

Piccola proprietà contadina - Agevolazioni tributarie - Agevolazioni fiscali in favore della piccola proprietà contadina ex art. 2 d.1. n. 194 del 2009 - Presupposti Iscrizione alla gestione previdenziale ed assistenziale dei coltivatori diretti presso l'Inps - Necessità - Conseguenze - Perdita volontaria del requisito nel quinquennio dall'acquisto - Decadenza dal beneficio - Fattispecie

In tema di agevolazioni fiscali in favore della piccola proprietà contadina, il presupposto della iscrizione del coltivatore diretto o dell'imprenditore agricolo professionale nella relativa gestione previdenziale ed assistenziale, previsto dall'art. 2 del d.l. n. 194 del 2009 (conv., con modif., dalla l. n. 25 del 2010), ai fini della concessione delle agevolazioni per gli atti traslativi a titolo oneroso di terreni agricoli, non ha natura di requisito "istantaneo" (che deve ricorrere solo al momento dell'acquisto agevolato) ma - avuto riguardo al disposto dell'art. 1647 c.c. coordinato con quello delle disposizioni delle leggi speciali in materia (da cui si desume che l'obbligo di iscrizione nella gestione previdenziale dei coltivatori diretti è subordinato allo svolgimento di tale attività con abitualità e prevalenza rispetto ad altre eventuali attività lavorative) - deve essere caratterizzato dalla permanenza nel tempo, sicché la cancellazione dell'iscrizione per effetto di una condotta volontaria (nella specie, acquisizione dello "status" di pensionato, di per sé non incompatibile con la qualifica di coltivatore diretto) comporta la perdita dell'agevolazione ove si verifichi entro un quinquennio dall'acquisto, conformemente a quanto espressamente sancito dall'art. 2, comma 4 bis, del d.lgs. $n$. 99 del 2004, per il coltivatore diretto che sia socio o amministratore "qualificante" della società agricola.

Cass. civ., Sez. V, Ordinanza, 12/02/2021, n. 3597

Acquisto compendio agricolo - imposta di registro e ipotecaria - misura fissaInizio modulo

Fine modulo

L'atto di compravendita di un compendio agricolo costituito da terreni e fabbricati funzionali all'attività agricola compresi quelli destinati ad uso ufficio dell'azienda agricola e agrituristica ed i fabbricati abitativi rurali sconta l'imposta di registro e ipotecaria in misura fissa in quanto ciò che rileva è il rapporto di pertinenzialità che viene a configurarsi in funzione dell'attività di impresa.

Cass. civ., Sez. V, Ordinanza, 10/02/2021, n. 3260

Piccola proprietà contadina - Agevolazioni tributarie - Agevolazioni "ex lege" n. 604 del 1954 - Esercizio del diritto di riscatto agrario - Affitto del fondo rustico entro il quinquennio dal suo acquisto - Conseguenze - Decadenza dal beneficio - Fondamento 
L'acquirente di un fondo rustico che abbia esercitato il diritto di riscatto agrario, avvalendosi delle agevolazioni fiscali relative all'acquisto della piccola proprietà contadina e che entro il quinquennio successivo affitti il bene a terzi, decade dal trattamento agevolativo, indipendentemente dal fatto che l'esercizio del diritto di riscatto comporti la sostituzione del riscattante nella posizione dell'originario acquirente con effetto retroattivo, essendo necessario, ai sensi dell'art. 7 della l. n. 604 del 1954, che egli provveda per cinque anni alla coltivazione diretta del fondo.

\section{PRELAZIONE E RISCATTO}

Cass. civ., Sez. III, 13/05/2021, n. 12894

Prelazione - denuntiatio - contenuto

In tema di prelazione agraria qualora la denuntiatio riguardi sia il fondo in affitto sia altri beni per $i$ quali il diritto di prelazione non sussiste ed indichi, però, separatamente il prezzo dei cespiti, il titolare della prelazione può manifestare la volontà di acquistare soltanto il bene in affitto $e$ non anche il bene estraneo, in tal modo rendendo effettiva la prelazione soltanto per il primo; $e$ in questo caso si avrà una manifestazione idonea a determinare gli effetti del positivo esercizio della prelazione, con quanto ne consegue. Se, invece, com'è accaduto nel caso di specie, l'offerta non indica i prezzi diversificati per i beni assoggettati a prelazione rispetto agli altri, l'eventuale accettazione non potrà ritenersi come valida manifestazione di volontà all'interno dell'esercizio del diritto di prelazione.

Cass. civ., Sez. III, Ordinanza, 16/03/2021, n. 7292ì

Prelazione - concorso tra più prelazionari - individuazione.

In presenza di una pluralità di coltivatori diretti proprietari di terreni diversi, tutti confinanti con il fondo rustico posto in vendita, a ciascuno dei medesimi spetta il diritto di prelazione e riscatto di cui all'art. 7, comma 2, n. 2), della l. n. 817 del 1971, e, ove si verifichi una situazione di conflittualità, per effetto dell'esercizio della prelazione o riscatto da parte di due o più dei predetti confinanti, la scelta del soggetto preferito è compito riservato al giudice del merito, che dovrà accordare prevalenza ad uno piuttosto che agli altri aspiranti alla prelazione, alla stregua della maggiore o minore attitudine a concretare la finalità perseguita dalla citata norma ed in applicazione, inoltre, degli specifici criteri preferenziali dettati dall'art.7 del d.los. n.228 del 2001.

\section{$\underline{\text { USI CIVICI }}$}

Cass. civ., Sez. Unite, Ordinanza, 26/03/2021, n. 8564

Usi civici - Giurisdizione del commissario regionale per la liquidazione degli usi civici - Contenuto - "qualitas soli" antecedente logico-giuridico della decisione Impugnazione di atto amministrativo - Giurisdizione dell'a.g.a. - Configurabilità - Limiti

La giurisdizione del commissario per la liquidazione degli usi civici sussiste ogniqualvolta la valutazione o l'accertamento della natura ed estensione del diritto di uso civico - cioè, la 
"qualitas soli" - si pongono come antecedente logico-giuridico della decisione; pertanto, in caso di impugnazione di atto amministrativo, la giurisdizione spetta al g.a. soltanto se le questioni dedotte sono dirette a censurare l'iter procedimentale, antecedentemente rispetto ad ogni indagine sulla qualità demaniale collettiva dei terreni. 